\title{
Propiedades fisicoquímicas de aceites provenientes de Salvia hispanica L., Linum usitatissinum y Plukenetia volubilis $\mathrm{L}$
}

\section{Physicochemical properties of oils from Salvia hispánica L., Linum usitatissinum and Plukenetia volubilis $\mathrm{L}$}

\author{
Eduard Vilcarromero Chavez ${ }^{1}$, Segundo Víctor Olivares Muñoz ${ }^{2}$
}

\section{RESUMEN}

La investigación tuvo por objetivo comparar las propiedades fisicoquímicas de aceites provenientes de Salvia hispánica L. "chía", Linum usitatissinum "linaza" y Plukenetia volubilis L. "sacha inchi”. La extracción del aceite de las semillas se realizó por método Soxhlet con arrastre de solvente, éter de petróleo y etanol. Los resultados fueron evaluados mediante análisis de varianza para determinar las probables diferencias significativas en las propiedades. En consecuencia, se determinó que el rendimiento en el aceite de chía fue de $23,7 \%$ usando etanol, en el de linaza fue de $26,8 \%$ y para sacha inchi fue de $34,4 \%$, usando éter de petróleo, referente a la densidad, los aceites muestran valores aproximados de $0,9 \mathrm{~g} / \mathrm{ml}$, el índice de refracción en los tres aceites en promedio fueron 1,48; los valores de saponificación reportados son mayores a 118,6 $\mathrm{mg} \mathrm{KOH} / \mathrm{g}$; y peróxido estuvieron entre 1,9 a 2,6 meq de $\mathrm{O}_{2} / \mathrm{kg}$ de aceite. En cuanto a la comparación del tipo de semilla los valores de saponificación y de peróxido no presentan valores estadísticamente diferentes, en el caso del solvente no presento efectos significativos sobre la densidad y el valor de peróxido.

Palabras clave: Aceite, chía, linaza, sacha inchi

\begin{abstract}
The objective of the research was to compare the physicochemical properties of oils from Chia, Linseed and Sacha inchi. The extraction of the oil from the seeds was carried out by the Soxhlet method with stripping of solvent, petroleum ether and ethanol. The results were evaluated by analysis of variance to determine the probable significant differences in the properties. Consequently, it was determined that the yield for chia oil was $23.7 \%$ using ethanol, for flaxseed it was $26.8 \%$ and for Sacha inchi it was 34.4\%, using petroleum ether, referring to the density, the oils show approximate values of $0.9 \mathrm{~g} / \mathrm{ml}$, the refractive index in the three oils on average were 1.48; the reported saponification values are greater than $118.6 \mathrm{mg} \mathrm{KOH} / \mathrm{g}$; and peroxide were between 1.9 to $2.6 \mathrm{meq}$ $\mathrm{O} 2$ / kg oil. Regarding the comparison of the type of seed, the saponification and peroxide values do not present statistically different values, in the case of the solvent, it did not present significant effects on the density and the peroxide value.
\end{abstract}

Keywords: Oil, chia, linseed, sacha inchi

\footnotetext{
${ }^{1}$ Bachiller en Ingeniería Agroindustrial de la Facultad de Ingeniería y Ciencias Agrarias de la Universidad Nacional Toribio Rodríguez de Mendoza de Amazonas. Correo electrónico: eduard.vilcarromero@gmail.com

${ }^{2}$ Docente auxiliar de la Facultad de Ingeniería Ciencias Agrarias de la Universidad Nacional Toribio Rodríguez de Mendoza de Amazonas;

Ingeniero Agroindustrial; con Maestría en Docencia y gestión universitaria. Correo electrónico: segundo.olivares@untrm.edu.pe
} 


\section{INTRODUCCIÓN}

En la actualidad existe una amplia gama de fuentes de aceites vegetales, además el consumo mundial está dominado por los aceites de palma, soja, colza y girasol. Sin embargo, en los últimos años con la creciente demanda de grasas y aceites se ha generado un desarrollo de especies de plantas prometedoras como fuente de aceites, los cuales contienen cantidades significativas de aceites y/o una alta proporción de ácidos grasos (Cheng et al., 2016 y Ixtaina et al., 2011).

Estos aceites están presentes en las organelas de granos oleaginosos, las cuales deben romperse para su obtención; este tipo de aceites es de un alto valor calorífico. La obtención se realiza por prensado mecánico o extracción con solvente. Cada proceso tiene características particulares; por un lado, la extracción por prensado está condicionado en el rendimiento ya que depende de la humedad, la cocción y la composición química. En la extracción con solventes genera poca pérdida de aceite en el proceso (Cheng et al., 2019; Durán et al., 2015; Mara y Barrera-Arellano, 2009; Tabio et al., 2017). De estos dos, Tabio et al. (2017) indican que la metodología más usada para extraer aceites es con solventes (Soxhlet).

En estudios previos, el Sacha inchi muestra un alto contenido de aceite $(41-54 \%)$ y tiene un alto contenido de ácidos grasos poliinsaturados (Niu et al., 2014; Rodríguez et al., 2015 y Zanqui et al., 2016). En el estudio de Paucar-Menacho et al. (2015) y Wang et al. (2018) indican que el aceite obtenido posee una densidad relativa de 0,91 , un índice de refracción de 1,47 y valor de saponificación está entre 185-193 mg KOH/g. Asimismo, el aceite proveniente de chía es una fuente importante de proteínas, fibra dietética, omega-3, alto contenido de ácido $\alpha$-linolénico y compuestos bioactivos con propiedades nutricionales (Guiotto, 2014 y Marineli et al., 2014). En cuanto a su extracción Ixtaina et al. (2011), determinan que el rendimiento del aceite es mejor con solvente, y la calidad de los aceites es influenciado por el proceso de extracción.

Otra fuente importante es la linaza en donde el aceite está en los cotiledones, que es rico en ácido palmítico, ácidos $\alpha$-linolénico, linoleico y oleico (Figuerola et al., 2008; Wang et al., 2017; Zettel y Hitzmann, 2018). El rendimiento es mayor por Soxhlet en aceite de semillas de linaza (Méndez y Ramos, 2017). Según Arias y López (2015) y Silva et al. (2013) presenta un bajo porcentaje de acidez y de peróxidos, y un índice de refracción relativamente elevado por su contenido de ácidos grasos insaturados. Además, presenta una acidez entre 0,5 y $0,8 \mathrm{mg} \mathrm{KOH} / \mathrm{g}$ de aceite y un índice de saponificación entre 189,6 y 191,5 mg KOH/g aceite.
Los antecedentes evidencian que la extracción por medio de solventes produce aceites de buena calidad y de un considerable rendimiento. Asimismo, las semillas de chía, linaza y sacha inchi son fuentes alternas de generación de aceites vegetales que pueden ser un importante producto en la región Amazonas. Por lo que en la investigación se realizó un estudio de las propiedades fisicoquímicas entre los aceites y los solventes a utilizar, debido que estas propiedades demuestran el grado en el que un conjunto de características inherentes cumple con la necesidad o expectativa establecida.

\section{MATERIAL Y MÉTODOS}

\subsection{Muestras recolectadas.}

Las semillas de chía, linaza y sacha inchi se compraron en el mercado central de Chachapoyas (01,5 kg de cada semilla); se seleccionó, homogenizó y envasó en recipientes herméticos.

\subsection{Diseño de la investigación.}

Se utilizó un diseño experimental para comparar tres tipos de aceites (chía, linaza y sacha inchi) extraídos por dos métodos (éter de petróleo y alcohol); en función de la evaluación de las propiedades de los aceites.

\subsection{Determinación de propiedades fisicoquímicas Humedad de las semillas}

Se realizó en estufa convencional a $105^{\circ} \mathrm{C}$ hasta peso constante (Michajluk et al., 2018) y se calculó mediante la siguiente ecuación:

$$
\text { Humedad (\%) }=\frac{M_{\text {inicial }}-M_{\text {final }}}{M_{\text {inicial }}}
$$

\section{Cenizas de las semillas}

Según la NTP 205.004 (Acosta y Torres, 2015); se colocó un crisol $\left(\mathrm{m}_{0}\right)$ en un desecador hasta que se enfríe, se pesó en balanza analítica. Se pesó $2 \mathrm{~g}$. de muestra homogeneizada $\left(\mathrm{m}_{1}\right)$. Se pre calcinó en placa calefactora, evitando que se inflame, se colocó en la mufla e incineró a $550^{\circ} \mathrm{C}$ por 8 horas. Se enfrió en desecador, se pesó (m2) y se calculó aplicando la ecuación:

$$
\% \text { Cenizas totales }=\frac{\left(\mathrm{m}_{2}-\mathrm{m}_{0}\right)}{\left(\mathrm{m}_{1}-\mathrm{m}_{0}\right)} \times 100
$$

\section{Extracción del aceite}

Se realizó por método Soxhlet con arrastre de solvente orgánico (Gutiérrez et al., 2014), procediendo a moler y pesar muestras de $100 \mathrm{~g}$, se colocó en la cámara Soxhlet, con $250 \mathrm{ml}$ de solvente por $6 \mathrm{~h}$. aproximadamente. El extracto se dejó reposar, se filtró y el aceite obtenido se conservó en refrigeración.

\section{Cálculo del rendimiento}

Para calcular el rendimiento se utilizó la ecuación: 


$$
\% \mathrm{R}=\frac{\text { Aceite }(\mathrm{ml})}{\text { Semilla }(\mathrm{gr})} \times 100
$$

\section{Determinación de la densidad}

Para la determinación de la densidad se usó el densímetro del laboratorio de tecnología agroindustrial de la UNTRM. La medición se expresó en $\mathrm{g} / \mathrm{ml}$.

\section{Determinación del índice de refracción}

Se utilizó el método normado por la AOCS 28 Cc-725 (Paucar-Menacho et al., 2015) con un refractómetro digital, para lo cual se colocó una gota en el prisma inferior, se ajustan los prismas, se dejó en reposo por un minuto. Se ajustó la luz para obtener una lectura clara, se cuadró el plano colocando la línea divisoria en el centro del cruce. En la escala de arriba se lee IR (valor de la refracción).

\section{Índice de acidez}

Se hizo uso del método de la IUPAC 2.201 (PaucarMenacho et al., 2015), lo cual se basa en la titulación directa; se pesó la muestra de aceite en un matraz y se añadió en una proporción de 1:10 (muestra: alcohol) alcohol etílico neutralizado y dos gotas de fenolftaleína. Se tituló con hidróxido de sodio $0,1 \mathrm{~N}$ hasta la aparición de un ligero color rosa y se procedió al cálculo con la siguiente formula:

$$
I \mathrm{~A}=\frac{\mathrm{V} \times \mathrm{N} \times 56.1}{\mathrm{~W}}
$$

Donde IA expresa los miligramos de $\mathrm{NaOH}$ requeridos para neutralizar los ácidos grasos libres (oleico); $\mathrm{V}$ es el volumen en $\mathrm{mL}$ de la solución $\mathrm{NaOH}$ utilizada; $\mathrm{N}$ es la concentración normal de $\mathrm{NaOH}$; y W el peso en gramos de la muestra de aceite.

\section{Índice de peróxido}

Se determinó siguiendo la técnica de la American Oil Chemists Society - AOCS (2010); en consecuencia se pesó la muestra en un matraz de $250 \mathrm{~mL}$ y se agregó (1:6) la solución 3: 2 de ácido acéticocloroformo. Se agitó hasta disolver, se agregó $0,5 \mathrm{ml}$ de solución saturada de KI con una pipeta, se dejó reposar exactamente 1 minuto y se adicionó agua destilada. Se colocó gradualmente tiosulfato de sodio $0,1 \mathrm{~N}$ hasta que el color amarillo de yodo desaparezca. Se añadió $2,0 \mathrm{ml}$ de solución indicadora de almidón. Se continuó con la titulación con agitación constante, especialmente cerca del punto final y se agregó la solución de tiosulfato gota a gota hasta que el color azul desaparezca. Posteriormente se realizó el cálculo del valor de peróxido con la siguiente formula:

$$
\mathrm{IP}=\frac{(\mathrm{S}-\mathrm{B}) \times \mathrm{N} \times 1000}{\text { Peso de la muestra (g) }}
$$

Donde IP es índice de peróxido (miliequivalentes peróxido / $1000 \mathrm{~g}$ muestra), B es el volumen de titulante, ( $\mathrm{ml}$ de blanco), $\mathrm{S}$ es el volumen de titulante (ml de muestra) y N es la normalidad de la solución de tiosulfato de sodio

\section{Índice de saponificación}

Se determinó con el método CD 3-25 de la (AOCS, 1993), en consecuencia se pesó $5 \mathrm{~g}$ de aceite en un matraz de $250 \mathrm{~mL}$, se añadió $50 \mathrm{~mL}$ de la solución etanólica de $\mathrm{KOH}$, se sometió a reflujo suavemente por 30 minutos aproximadamente. Se enfrió y tituló el exceso de álcali con $\mathrm{HCl} \quad 0,5 \mathrm{~N}$. Además, se hace una determinación en blanco. Luego se calculó con la siguiente ecuación:

$$
\text { IS }=\frac{\left(\mathrm{V}_{1}-\mathrm{V}_{2}\right) \times \mathrm{N} \times 56,1 \mathrm{~g} / \mathrm{eq}}{\mathrm{G}}
$$

Dónde: $\mathrm{V} 1: \mathrm{ml}$ de $\mathrm{HCl}$ gastados en la valoración del ensayo en blanco; V2: $\mathrm{ml}$ de $\mathrm{HCl}$ gastados en la valoración de la muestra; N: Normalidad de la solución de $\mathrm{HCl}$; G: Peso de la muestra (g)

\subsection{Análisis de datos}

Los resultados fueron evaluados mediante el cálculo de medias y desviaciones estándar. Además, se recurrió a la prueba de ANOVA para determinar las posibles diferencias significativas en las propiedades fisicoquímicas entre los aceites investigados. Las diferencias se consideraron estadísticamente significativas al nivel de $p<0,05$.

\section{RESULTADOS}

\section{Propiedades fisicoquímicas de los aceites de chía,} linaza y sacha inchi

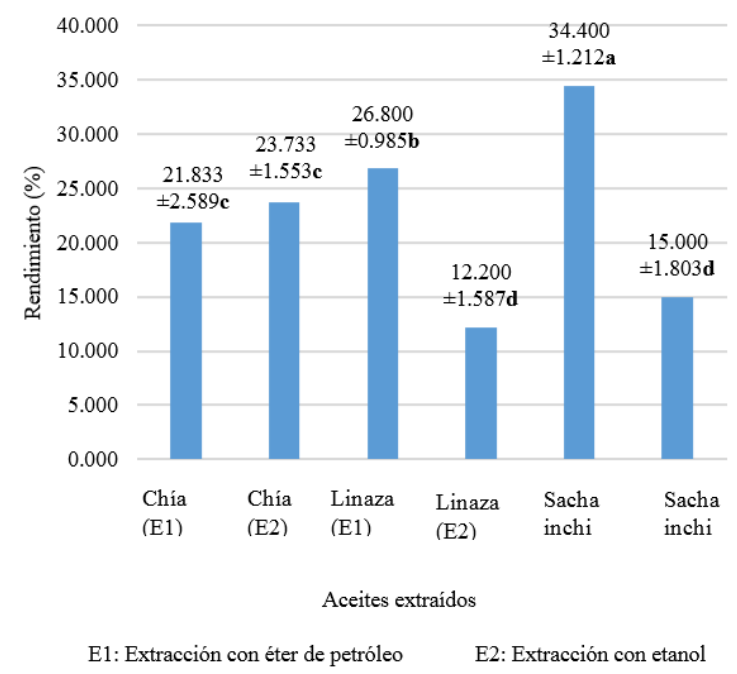

Figura 1. Rendimiento de los aceite

En la figura 1, se observa que el rendimiento mayor de aceite es de las semillas de sacha inchi extraídas por éter de petróleo es mayor en comparación al resto de semillas

El rendimiento de los aceites varió de $12,2 \pm 1,58 \mathrm{~d}$ a $34,4 \% \pm 1,21 \mathrm{a}$, de otro lado se observa en la figura 1 
que el aceite de chía extraído por etanol se obtuvo un mayor rendimiento en comparación al éter de petróleo.

Asimismo, la figura muestra la formación de cuatro grupos de media estadísticamente distintos, de los cuales en el caso de la chía el rendimiento es igual en ambas extracciones.

\section{Tabla 1. Densidad de los aceites obtenidos}

\begin{tabular}{ll}
\hline Aceite & Densidad $(\mathrm{g} / \mathrm{ml})$ \\
\hline Chía (E1) & $0,849 \pm 0,026 \mathbf{b}$ \\
Chía (E2) & $0,947 \pm 0,009 \mathbf{a}$ \\
Linaza (E1) & $0,868 \pm 0,058 \mathbf{b}$ \\
Linaza (E2) & $0,952 \pm 0,036 \mathbf{a}$ \\
Sacha inchi (E1) & $0,891 \pm 0,002 \mathbf{b}$ \\
Sacha inchi (E2) & $0,771 \pm 0,025 \mathbf{c}$
\end{tabular}

En la tabla 1 se observa que la densidad de los aceites de chía y linaza extraída mediante alcohol es superior $(>0,9 \mathrm{~g} / \mathrm{ml})$ en comparación al extraído con éter de petróleo; de otro lado, el aceite de sacha inchi presenta una baja densidad. Además, se obtuvo tres grupos de media en cuanto a la densidad que son diferentes entre sí.

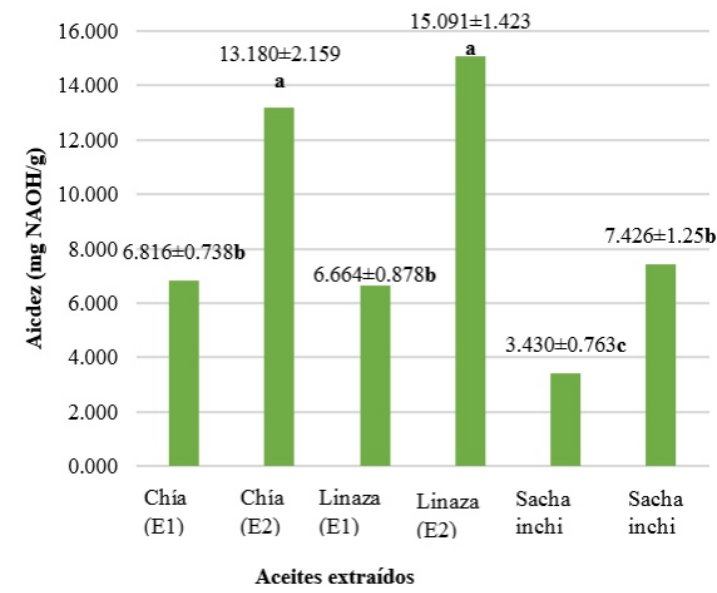

Figura 2. Índices de acidez de los aceites de chía, linaza y sacha inchi

En la figura 2, referente a la acidez se observa que usando éter de petróleo en la extracción de aceites se obtiene un menor valor en todas las semillas estudiadas, observando que el sacha inchi posee un menor índice de acidez (3,43 $\mathrm{mg}$ de $\mathrm{NAOH} / \mathrm{g})$. De otro lado, mediante la prueba de Fisher se observa que existen tres grupos de medias en los valores de acidez obtenidos, donde se observa que el aceite obtenido de chía y linaza por medio de etanol presentan mayores índices en comparación al resto.

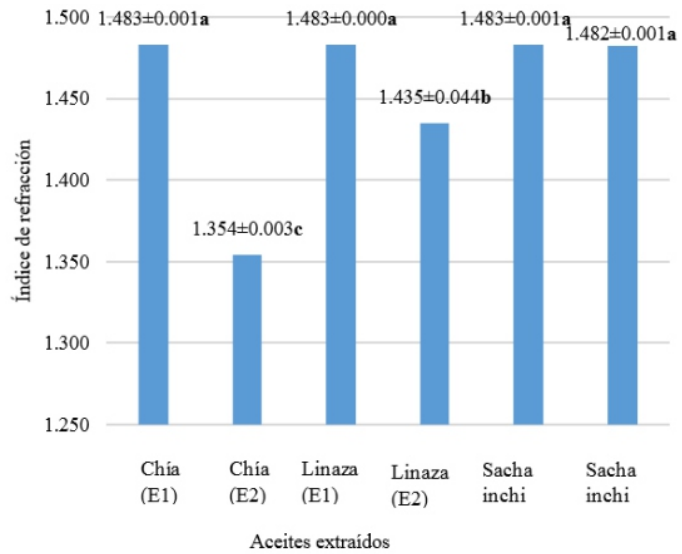

Figura 3. Índices de refracción de los aceites

Respecto al índice de refracción, en la figura 3 se observa que el valor estuvo alrededor de 1,48, además, los aceites de las tres semillas extraídas por éter de petróleo y el aceite de sacha inchi por etanol presentan promedios similares.

Tabla 2. Índice de saponificación y de peróxido de los aceites

\begin{tabular}{ccc}
\hline Aceite & $\begin{array}{c}\text { Índice de } \\
\text { saponificación }\end{array}$ & $\begin{array}{c}\text { Índice de } \\
\text { peróxido }\end{array}$ \\
\hline Chía (E1) & $120,691 \pm 0,916 \mathbf{a}$ & $1,988 \pm 0,024^{\mathbf{a}}$ \\
Chía (E2) & $107,830 \pm 2,368 \mathbf{b}$ & $3,339 \pm 1,159^{\mathbf{a}}$ \\
Linaza (E1) & $118,610 \pm 0,305 \mathbf{a}$ & $2,661 \pm 1,145^{\mathrm{a}}$ \\
Linaza (E2) & $107,636 \pm 6,213 \mathbf{b}$ & $2,003 \pm 0,005^{\mathbf{a}}$ \\
Sacha inchi (E1) & $121,883 \pm 3,105 \mathbf{a}$ & $2,671 \pm 1,151^{\mathrm{a}}$ \\
& & \\
Sacha inchi (E2) & $111,082 \pm 5,141 \mathbf{b}$ & $2,668 \pm 1,154^{\mathbf{a}}$ \\
\hline
\end{tabular}

Algunas de las propiedades fisicoquímicas importantes como índice de saponificación y peróxido; en la tabla 3 se observa que los aceites obtenidos de las semillas utilizando éter de petróleo tienen valores de saponificación mayores a $111 \mathrm{mg}$ $\mathrm{KOH} / \mathrm{g}$. En cuanto al índice de peróxido, se obtuvo que el aceite de chía presenta un mayor valor de peróxido $(3,339 \pm 1,159 \mathrm{meq}$ de $\mathrm{O} 2 / \mathrm{kg}$ de aceite $)$ en comparación a los aceites de linaza y sacha inchi, sin embargo, la prueba de comparación de medias reporta que no existe diferencia estadísticamente suficiente en la peroxidación de aceites.

Comparación de los aceites de chía, linaza y sacha inchi obtenidos por diferentes solventes

En la tabla 4, se observa que existe diferencia significativa del rendimiento, índice de acidez y de refracción debido al solvente y las semillas; en cuanto a la densidad existe efecto de la semilla sobre la densidad; y para el valor de saponificación existe efecto del solvente sobre el contenido de esta 
propiedad.

Tabla 3. Resumen del ANOVA de las propiedades fisicoquímicas de los aceites.

\begin{tabular}{lc|c|c|c|c|c}
\hline & $\mathbf{R}$ & $\mathbf{D}$ & $\mathbf{I A}$ & $\mathbf{I} \mathbf{R}$ & $\mathbf{I S}$ & $\mathbf{I P}$ \\
\hline \multicolumn{1}{c}{ Fuente } & Valor & Valor & Valor & Valor & Valor & Valor \\
& $\mathbf{p}$ & $\mathbf{p}$ & $\mathbf{p}$ & $\mathbf{p}$ & $\mathbf{p}$ & $\mathbf{p}$ \\
\hline Modelo & 0,000 & 0,000 & 0,000 & 0,000 & 0,001 & 0,531 \\
Lineal & 0,000 & 0,004 & 0,000 & 0,000 & 0,000 & 0,854 \\
Fuente & 0,001 & 0,002 & 0,000 & 0,000 & 0,310 & 0,781 \\
Solvente & 0,000 & 0,182 & 0,000 & 0,000 & 0,000 & 0,614 \\
Interacciones & 0,000 & 0,000 & 0,038 & 0,000 & 0,867 & 0,211 \\
de 2 términos & & & & & & \\
Fuente & 0,000 & 0,000 & 0,038 & 0,000 & 0,867 & 0,211 \\
*Solvente & & & & & & \\
\hline R-Cuad. & 96,56 & 84,97 & 93,54 & 91,46 & 79,73 & 26,51 \\
& $\%$ & $\%$ & $\%$ & $\%$ & $\%$ & $\%$ \\
\hline
\end{tabular}

$\mathrm{R}$ : Rendimiento

D: Densidad

IA: Índice de acidez

IR: Índice de refracción

IS: Índice de saponificación

IP: Índice de peróxido

De otro lado, el valor de peróxido no tiene diferencias significativas, es decir, la fuente y el solvente no tienen efecto sobre el contenido lo cual se ve comprobado a través de una baja relación (R2 bajo).

\section{DISCUSIÓN}

Los resultados experimentales para el rendimiento, en el aceite de chía fue de 23,7 $71,553 \%$ usando etanol, este resultado es próximo a lo obtenido por Guiotto (2014) que indica que la semilla tiene un contenido de aceite alrededor del $33 \%$.

En el aceite de linaza, Figuerola et al. (2008) y Méndez y Ramos (2017) indican que el método Soxhlet se obtiene un mayor rendimiento entre el 35 a $43 \%$, el rendimiento obtenido en el estudio fue de $26,8 \pm 0,985 \%$ un valor próximo respecto a 10 mencionado por los autores, en el caso del solvente guarda concordancia con lo obtenido por Méndez y Ramos (2017) indicando que con éter de petróleo se obtiene una mayor producción de aceite de las semillas. Los resultados muestran que el rendimiento del aceite de sacha inchi fue de 34,4 $\pm 1,212 \%$ usando éter de petróleo, similar a lo obtenido por Chirinos (2015), que fue $35,4 \%$ con el mismo solvente.

Referente a la densidad, los aceites muestran valores aproximados de $0,9 \mathrm{~g} / \mathrm{ml}$. En el caso del aceite de chía y linaza mediante la prueba de Fisher se observa que usando etanol se obtienen un mayor valor respecto al resto de las muestras. De otro lado, en el aceite de linaza el valor obtenido $(0,95 \pm 0,036 \mathrm{~g} / \mathrm{ml})$ guarda relación con lo hallado por Arias y López (2015) y Silva et al. (2013) los cuales indican que el aceite extraído por prensado de linaza posee una densidad entre 0,931 y $0,94 \mathrm{~g} / \mathrm{ml}$, con lo cual se puede deducir que el tipo de extracción (solvente o prensado) no influye en la densidad del aceite.

En cuanto al índice de acidez, a través de la extracción con éter de petróleo en las semillas de chía se obtuvo un menor valor $(6,8 \pm 0,78 \mathrm{mg} \mathrm{NAOH} / \mathrm{g}) \mathrm{de}$ acidez, este valor es próximo a lo reportado por Timilsena et al. (2017) indicando que este aceite posee menores cantidades de ácidos grasos libres y oxidación. En el caso del aceite de linaza, el valor obtenido en $\mathrm{mg} \mathrm{NAOH} / \mathrm{g}$ en contraposición con los otros resultados reportados por Silva et al. (2013) cuyos índice de acidez fueron entre 0,588 y $0,811 \mathrm{mg}$ $\mathrm{KOH} / \mathrm{g}$ de aceite si bien las unidades de medida son distintas la tendencia es que estos valores deben ser bajos. Para el aceite de sacha inchi el menor valor de acidez fue de 3,4 $\pm 0,763$ (extracción con éter de petróleo), similar a los reportado por Chasquibol et al. (Citado en Wang et al., 2018).

Los índices de refracción en los tres aceites valores promedio fue de 1,48, en el aceite de chía y linaza extraído con etanol se obtiene un menor índice en comparación con el éter de petróleo, este valor refractivo incrementa a medida que aumenta el número de dobles enlaces. Los valores de refracción reportados son similares según lo mencionado por Paucar et al. (2015), Silva et al. (2013), Timilsena et al. (2017) y S. Wang et al. (2018) los cuales determinan que estos aceites poseen un índice de refracción de 1,48.

Los valores de saponificación reportados en el presente estudio de los aceites obtenidos de las semillas utilizando éter de petróleo tienen valores de saponificación mayores a 118,6 $\pm 0,305 \mathrm{mg} \mathrm{KOH} / \mathrm{g}$ que guardan proximidad con los valores determinados por Timilsena et al. (2017), Silva et al. (2013) y Gutiérrez et al. (2014) los cuales establecen un rango de saponificación entre 185,2 a 191,6. En el caso de los valores de peróxido se obtuvieron valores entre 1,9 a 2,6 meq de $\mathrm{O} 2 / \mathrm{kg}$ de aceite, los cuales guardan relación con lo reportado por Timilsena et al. (2017) cuyo valor fue de 4,33 meqO2/kg de aceite de chía, y Silva et al. (2013) cuyo índice de peróxido estuvo entre $0,256-1,12 \mathrm{meqO} 2 / \mathrm{kg}$ de aceite de linaza.

En cuanto a la comparación de las formas de extracción de los aceites, se observa que en cuanto al tipo de semilla los valores de saponificación y de peróxido no presentan valores estadísticamente diferentes, en el caso del solvente no presentó efectos significativos sobre la densidad y el valor de peróxido obtenido. Se reporta que el índice de peróxido no se vio afectado por los tratamientos aplicados.

\section{CONCLUSIONES}

Las semillas de chía, linaza y sacha inchi fueron caracterizados por el rendimiento, en el caso del aceite de chía fue de $23,7 \%$ usando etanol, el aceite de linaza fue de $26.8 \%$, y el rendimiento del aceite de sacha inchi fue de 34,4\% usando éter de petróleo en estas dos últimas semillas. 
Las densidades de los aceites son aproximadamente de $0,9 \mathrm{~g} / \mathrm{ml}$, los valores de refracción, acidez, peróxido y saponificación demuestra la calidad de los aceites obtenidos. En cuanto a la comparación del tipo de semilla los valores de saponificación y de peróxido no presentan valores estadísticamente diferentes, en el caso del solvente no presento efectos significativos sobre la densidad y el valor de peróxido obtenido

\section{REFERENCIAS BIBLIOGRÁFICAS}

Acosta, I. X., \& Torres, G. (2015). Extracción de aceite Chía (Salvia hispánica L.) por el método de prensado continuo (expeller) y discontinuo (hidráulico) de las regiones del Cusco y Arequipa. Universidad Nacional San Agustín.http://repositorio.unsa.edu.pe/bitstrea $\mathrm{m} / \mathrm{h}$ a n d l e / U N S A / 238 / B 2 - M 18426.pdf? sequence $=1 \&$ is Allowed $=$

American Oil Chemists Society - AOCS. (1993). AOCS Official Method Cd Official Methods and Recomended Practices of the $\operatorname{AOCS}(p .1200)$.

American Oil Chemists Society - AOCS. (2010). AOCS SURPLUS Method Cd 8-53. Declared Surplus 2003. Peroxide Value-Acetic AcidChloroform Method. En Official Methods and Recommended Practices of AOCS (Quinceava). AOCS.

Arias, J., \& López, N. (2015). Estabilidad oxidativa y perfil de ácidos grasos del aceite de semilla de lino (Linum usitatissisum) procedentes de Corongo y Otuzco, extraído por prensado en frío [Tesis de Grado, Universidad Nacional del Santa]. http://repositorio.uns.edu.pe/bitstream/ha n d 1 e/UNS/1987/30733.pdf?sequence $=1$ \&isA llo wed $=\mathrm{y}$

Cheng, M.-H., Dien, B. S., \& Singh, V. (2019). Economics of plant oil recovery: $A$ review. Biocatalysis and Agricultural Biotechnology, 18,101056. https://doi.org/10.1016/j.bcab.2019.101056

Cheng, W.-Y., Haque Akanda, J. M., \& Nyam, K.- L. (2016). Kenaf seed oil: A potential new source of edible oil. Trends in Food Science \& Technology, 52, 5765.https://doi.org/10.1016/j.tifs.2016.03.0 14

Durán, S., Torres, J., \& Sanhueza, J. (2015).
Aceites vegetales de uso frecuente en Sudamérica: Características y propiedades. Nutrición Hospitalaria, 32(1), 11-19. https://doi.org/10.3305/nh.2015.32.1.8874

Figuerola, F., Muñoz, O., \& Estévez, A. M. (2008). La Linaza como fuente de compuestos bioactivos para la elaboración de alimentos. Agro Sur, 36 (2), $49-58$. https://doi.org/10.4206/agrosur.2008.v36n2-01

Guiotto, E. N. (2014). Aplicación de subproductos (Helianthus annuss L.) en alimentos [Tesis de Doctorado, Universidad Nacional De la Plata].http://sedici.unlp.edu.ar/handle/1091 5/34268

Gutiérrez, R., Ramírez, M. L., Vega, S., Fontecha, J., Rodríguez, L. M., \& Escobar, A. (2014). Contenido de ácidos grasos en semillas de chía (Salvia hispanica L.) cultivadas en cuatro estados de México. Revista Cubana de Plantas Medicinales, 19 (3), 199 207.http://scielo.sld.cu/scielo.php?script $=$ s c i_a b s tract \& p id = S 1028 47962014000300008 \& $1 \mathrm{ng}$ $=\mathrm{es} \& \mathrm{nrm}=\mathrm{iso} \& \mathrm{tlng}=\mathrm{es}$

Ixtaina, V. Y., Martínez, M. L., Spotorno, V., Mateo, C. M., Maestri, D. M., Diehl, B. W. K., Nolasco, S. M., \& Tomás, M. C. (2011). Characterization of chia seed oils obtained by pressing and solvent extraction. Journal of Food Composition and Analysis, 24(2), 166174.https://doi.org/10.1016/j.jfca.2010.08. 006

Mara, J., \& Barrera-Arellano, D. (2009). Temas Selectos en Aceites y Grasas: Vol. I (Primera). Blucher.

Marineli, R. da S., Moraes, É. A., Lenquiste, S. A., Godoy, A. T., Eberlin, M. N., \& Maróstica Jr, M. R. (2014). Chemical characterization and antioxidant potential of Chilean chia seeds and oil (Salvia hispanica L.). LWT - Food Science and Technology, 59(2), 1304-1310. https://doi.org/10.1016/j.lwt.2014.04.014

Méndez, D., \& Ramos, C. (2017). Comparación de métodos de extracción del aceite de la semilla de linaza (Linum usitatissinum). Revista Facultad de Ciencias Básicas. Momentos de C i e n c i a, 1 ( 1 ) . https://es.scribd.com/document/34394896 1/a ceite-de-linaza 
Michajluk, B. J., Piris, P. A., Merele S. B. (2018). Seeds of Salvia hispanica L., "chia» as a source of macronutrients, dietary fiber and minerals. Investigación Agraria, 20(1), 74-77. https://doi.org/10.18004/investig.agrar.20 18.junio.74-77

Niu, L., Li, J., Chen, M.-S., \& Xu, Z.-F. (2014). Determination of oil contents in Sacha inchi (Plukenetia volubilis) seeds at different developmental stages by two methods: Soxhlet extraction and time-domain nuclear magnetic resonance. Industrial Crops and P r o d u c t s , 56, $187-190$. https://doi.org/10.1016/j.indcrop.2014.03.007

Paucar, L. M., Salvador, R., Guillén, J., Cap, J., \& Moreno, C. (2015). Comparative study of physical-chemical features of sacha inchi oil (Plukenetia volubilis l.), olive oil (Olea europaea) and fish oil. Scientia a gropecuaria, 279 - 290 https://doi.org/10.17268/sci.agropecu.2015.0 4.05

Rodríguez, G., Villanueva, E., Glorio, P., \& Baquerizo, M. (2015). Oxidative stability and estimate of the shelf life of sacha inchi (Plukenetia volubilis L.) oil. Scientia a gropecuaria, 155 - 163. https://doi.org/10.17268/sci.agropecu.2015. 03.02

Silva, M., Gallardo, G., \& Pascual, G. (2013). Caracterización físico-química del Aceite de Linaza (Linum usitatissimum l.) del Departamento Cajamarca, Perú. Infinitum, 3 (1), $45-57$. http://revistas.unjfsc.edu.pe/index.php/INFI NITUM/article/download/382/351

Tabio, D., Díaz, Y., Rondón, M., Fernández, E., \& origen vegetal. Universidad Tecnológica de La Habana "José Antonio Echevarría». http://rgdoi.net/10.13140/RG.2.2.11047.552 01

Timilsena, Y. P., Vongsvivut, J., Adhikari, R., \& Adhikari, B. (2017). Physicochemical and thermal characteristics of Australian chia seed oil. Food Chemistry, 228, 394-402. https://doi.org/10.1016/j.foodchem.2017.02. 021

Wang, H., Wang, J., Qiu, C., Ye, Y., Guo, X., Chen, G., Li, T., Wang, Y., Fu, X., \& Liu, R. H. (2017).
Comparison of phytochemical profiles and health benefits in fiber and oil flaxseeds (Linum usitatissimum L.). Food Chemistry, 214, 227 $2 \quad 3 \quad 3$ https://doi.org/10.1016/j.foodchem.2016.07.07 5

Wang, S., Zhu, F., \& Kakuda, Y. (2018). Sacha inchi ( Plukenetia volubilis L.): Nutritional composition, biological activity, and uses. Food Che mistry, $265,316-328$. https://doi.org/10.1016/j.foodchem.2018.05.05 5

Zanqui, A. B., da Silva, C. M., de Morais, D. R., Santos, J. M., Ribeiro, S. A. O., Eberlin, M. N., Cardozo-Filho, L., Visentainer, J. V., Gomes, S. T. M., \& Matsushita, M. (2016). Sacha inchi (Plukenetia volubilis L.) oil composition varies with changes in temperature and pressure in subcritical extraction with n-propane. Industrial Crops and Products,

87, $\begin{array}{lllll}6 & 4 & - & 7 & 0\end{array}$. https://doi.org/10.1016/j.indcrop.2016.04.029

Zettel, V., \& Hitzmann, B. (2018). Applications of chia (Salvia hispanica L.) in food products. Trends in Food Science \& Technology, 80,4350. 